\section{Uso recente de álcool, tabaco e outras drogas entre estudantes adolescentes trabalhadores e não trabalhadores}

\section{Recent use of alcohol, tobacco, and other drugs among working and nonworking adolescents}

\author{
Delma P. Oliveira de Souza ${ }^{1,2}$ \\ Dartiu Xavier da Silveira Filho' \\ 'Programa de Pós-Graduação em Psiquiatria da Universidade Federal de São \\ Paulo \\ ${ }^{2}$ Instituto de Saúde Coletiva, Universidade Federal de Mato Grosso
}

Projeto financiado pela Fundação de Amparo à Pesquisa do Estado de Mato Grosso (FAPEMAT Processo n. 064/CAP/98). Bolsista do Programa Institucional de Capacitação Docente e Técnico Administrativo/CAPES/UFMT.

Correspondência: Delma P. Oliveira de Souza. Rua Safira, n 71 Bosque da Saúde - Cuiabá,MT - CEP 78008-300. E-mail: souzadpo@terra.com.br

\section{Resumo}

Foi realizado um estudo transversal, de base populacional, para determinar a prevalência do uso recente de substâncias psicoativas em uma amostra probabilística de 798 adolescentes trabalhadores e de 1.493 não trabalhadores, matriculados na rede estadual de educação básica da área urbana de Cuiabá, Brasil. Um questionário de autopreenchimento foi aplicado em sala de aula, indagando sobre aspectos sociodemográficas, sociais e o consumo de substâncias psicoativas. Definiu-se uso recente como consumo realizado uma ou mais vezes nos trinta dias que antecederam à entrevista. A prevalência na amostra do uso recente de álcool, tabaco e outras drogas foi de $37,4 \%, 9,5 \%$ e $8,4 \%$, respectivamente, sendo mais elevada entre os adolescentes trabalhadores do que entre os não trabalhadores $(47,4 \%$ versus $32,1 \%$ $\mathrm{RO}=1,91$, IC95\% 1,60-2,28; 13,6\% versus $7,3 \%$ RO $=1,98$, IC95\% 1,49-2,63; 11,1\% versus $6,9 \% \mathrm{RO}=1,68$, IC95\% 1,24-2,26). $\mathrm{Na}$ análise de regressão logística, o uso recente de álcool, tabaco e outras drogas manteve-se associado aos trabalhadores da faixa etária de 15-20 anos, do sexo masculino e baixo nível socioeconômico (C+D+E). Conclui-se que os resultados indicam que o uso recente de álcool, tabaco e outras drogas foi diferente entre os trabalhadores em comparação com os não trabalhadores, sugerindo que esses resultados podem orientar as ações articuladas de prevenção e tratamento à população adolescente.

Palavras-chave: Trabalho. Estudante Adolescente. Substâncias Psicoativas. Epidemiologia. Saúde Pública. 


\section{Abstract}

We carried out a cross-sectional, population-based study comparing the prevalence of recent psychoactive substance use in a probabilistic sample of 798 adolescent students that were also workers and of 1,493 that did not work. Students were enrolled in state Basic Education schools in the urban area of Cuiabá, Brazil. A selfadministered questionnaire was completed by subjects in the classroom. This questionnaire included items on sociodemographic and school-related variables, as well as on the consumption of psychoactive substances. Recent use was defined as once or more in the 30 days prior to the interview. Prevalence in the sample of recent use of alcohol, tobacco, and other drugs was $37.4 \%, 9.5 \%$, and $8.4 \%$, respectively, and was higher among workers than among non-workers $(\mathbf{4 7 . 4 \%}$ vs. $\mathbf{3 2 . 1 \%}$, OR = 1.91, 95\%CI 1.60-2.28; $\mathbf{1 3 . 6 \%}$ vs. $\mathbf{7 . 3 \%}, \mathrm{OR}=1.9895 \%$ CI $1.49-2.63 ; \mathbf{1 1 . 1 \%}$ vs. $6.9 \% \mathrm{OR}=1.68,95 \% \mathrm{CI} 1.24-2.26)$. After logistic regression analysis, use of alcohol, tobacco, and other drugs remained associated with working adolescents in the 1520 years age group, males, and from $\mathrm{C}+\mathrm{D}+\mathrm{E}$ social classes. We conclude that the recent use of psychoactive substances differed between working and non-working students. These results may support public health and education policies regarding the implementation of concatenated preventive and therapeutic measures targeting the adolescent population.

Keywords: Epidemiology. Work. Psychoactive Substances. Adolescent Student. Public Health.

\section{Introdução}

A Organização Internacional do Trabalho - OIT, estabelece uma estimativa mundial de 352 milhões de crianças menores de 17 anos vinculadas ao trabalho (ILO, 2002) ${ }^{1}$. A magnitude desta estimativa e a importância da saúde física e psicossocial deste grupo populacional tornam a questão do trabalho infanto-juvenil uma prioridade na agenda das políticas públicas. No Brasil existem crianças e adolescentes que, além de exercerem uma atividade laborativa, estão matriculados na rede de Educação Básica, compreendida pelo Ensino Fundamental, com clientela de 7 a 14 anos de idade, e pelo Ensino Médio, com alunos de 15 a 17 anos de idade, conforme previsto na Lei de Diretrizes e Bases de 1996. Estas faixas etárias referem-se ao início e ao término da escolarização, desconsiderando-se aqueles em defasagem escolar. Embora a legislação brasileira restrinja o trabalho deste grupo populacional, ele existe no país e suas características de inserção variam entre as regiões, em relação ao sexo, área urbana e grupos etários.

O trabalho infanto-juvenil no Brasil encontra suas raízes e características na própria história brasileira. Em termos populacionais, verificou-se no país um intenso crescimento nas ultimas décadas. De 70 milhões de habitantes na década de 60 para 147 milhões na década de 80 , entre os quais 44 milhões eram crianças e adolescentes entre 5 e 17 anos de idade. Nos anos 90 inicia-se uma desaceleração do crescimento populacional e uma diminuição da taxa de crianças e adolescentes em relação ao total da população. A Pesquisa Nacional por Amostragem por Domicílios - PNAD de 2001 revelou que, das 5,4 milhões de crianças e adolescentes trabalhadores, 4,4 milhões também estudam, enquanto 1 milhão apenas trabalha. Entre os que estudam e trabalham, $30 \%$ tem uma jornada semanal de 40 horas, exceto nos Estados de São Paulo, Rondônia e Mato Grosso, onde crianças e 
adolescentes trabalham mais de 40 horas semanais ${ }^{2}$.

No Brasil, há variação na carga horária de trabalho semanal de crianças e adolescentes em função da idade e da atividade desempenhada. Entre o grupo com menos de 10 anos predomina uma carga horária de 20 horas (4 horas diárias), chegando a 40 ou mais horas semanais para o grupo de 17 anos de idade $^{3}$.

Embora certos segmentos da sociedade considerem o trabalho uma fonte de benefícios para o jovem, através da elevação da auto-estima, responsabilidade pessoal, autonomia e preparação para a vida adulta, é sabido que o cansaço decorrente do trabalho precoce reduz a capacidade de concentração e que o uso de substâncias psicoativas provoca baixos índices de freqüência escolar, assim como altos índices de repetência e distúrbios do comportamento. Alem disso, as seqüelas referentes às doenças do trabalho de adolescentes aparecem, em sua maioria, na fase adulta, dificultando o desenvolvimento de políticas de prevenção para esta população ${ }^{4}$.

Com relação à questão do uso de substâncias psicoativas entre os adolescentes, existem vários estudos realizados no âmbito nacional ${ }^{5-9}$ e levantamentos epidemiológicos realizados com estudantes a partir de 10 anos de idade do Ensino Fundamental e Médio de escolas públicas, desde a década de 80, pelo Centro Brasileiro de Informações sobre Drogas Psicotrópicas, CEBRID $^{10}$. Todavia, no Brasil, poucos são os estudos que associam a questão do estudante adolescente trabalhador e não trabalhador com o uso de substâncias pasicoativas.

O objetivo deste estudo é comparar a prevalência do uso recente de álcool, tabaco e outras drogas entre estudantes adolescentes trabalhadores e não trabalhadores, e verificar sua associação com fatores sociodemográficos, escolares e de saúde, visando o aprofundamento científico da questão para subsidiar as políticas públicas de prevenção e tratamento.

\section{Método}

Tipo de estudo, local e população

Este estudo analítico de corte transversal é constituído de estudantes adolescentes trabalhadores e não -trabalhadores, matriculados no ano de 1998 em escolas da rede estadual de Ensino Fundamental e Médio do município de Cuiabá, capital do Estado de Mato Grosso. Na época do estudo, o município contava com uma população de 483.346 habitantes, entre os quais 96.284 na faixa etária de 10 a 19 anos. O município possui localização estratégica no que concerne à ligação das regiões Nordeste, Sudeste e Sul com o Norte do país. A localização e o crescimento socioeconômico, a partir da década de 70, fizeram com que Cuiabá passasse a apresentar problemas típicos de metropolização, entre eles o consumo de substâncias psicoativas na sua população jovem ${ }^{11}$, contando com poucas políticas de prevenção, tratamento e pesquisas para a questão.

Para esta investigação definiu-se como elegível ser matriculado na rede estadual de ensino e estar na faixa etária de 10 a 20 anos. Este ponto de corte teve por base os princípios da Organização Mundial de Saúde - OMS sobre delimitação de início e fim da adolescência, o contexto educacional e o estudo de Outeiral ${ }^{12}$, que considera a adolescência constituída de três fases: a primeira, de 10 a 14 anos, a segunda de 14 a 17 anos, e a terceira de 18 a 20 anos. Esta categorização enquadra-se no início e término da escolaridade da população em estudo, conforme a Lei de Diretrizes e Bases da Educação Nacional.

Para a inclusão no grupo que trabalha, considerou-se aquele que afirmou possuir um vínculo empregatício, trabalhando regularmente, assim como todo estudante na situação de desempregado nos últimos doze meses, que fez algum esforço na busca de remuneracão através de "bicos" ou engajamento no mercado formal ou informal. O grupo que não trabalha foi constituído dos estudantes adolescentes que 
afirmaram "não trabalhar, apenas estudar”, nunca exerceram atividades remuneradas, não fazem parte da economia ativa ou recebem mesada e são mantidos por seus responsáveis, embora exerçam dispêndio físico em trabalhos domésticos ou ajudem os pais ou parentes em atividades lucrativas sem receber remuneração ${ }^{13}$.

\section{A amostra}

Com base no objetivo deste estudo, que foi comparar o uso recente de substâncias psicoativas entre estudantes adolescentes trabalhadores e não-trabalhadores, o sorteio buscou uma amostragem representativa por conglomerados (escolas) e estratificada (estratos representam modalidades de ensino da Educação Básica ministradas nas escolas: estrato 1 - Ensino Fundamental; estrato 2 - Ensino Médio; estrato 3 Ensino Fundamental e Médio; estrato 4 Educação de Jovens e Adultos), obtida em dois estágios: no primeiro, foram sorteadas as escolas, no segundo, as turmas ${ }^{10,14}$.

Para a estimativa do tamanho mínimo de amostra, para uso recente, considerou-se:

- um erro alfa de 0,05 ;

- um erro beta de 0,20 ;

- uma prevalência de exposição ao trabalho para $25,0 \%$ dos estudantes;

- uma prevalência de uso de drogas recente, entre os estudantes que não trabalham, de 5,0\%;

- um efeito de desenho de 1,8\% pelo fato de o sorteio vir a ser feito por turmas e não aleatoriamente.

Foram incluídos, ainda, $10,0 \%$ para as perdas. Com esses procedimentos, a amostra mínima requerida para compor o grupo de estudantes adolescente trabalhadores seria de 618, e de 1.854 para não trabalhadores, totalizando 2.472 estudantes adolescentes.

\section{0 instrumento}

Empregou-se um questionário estruturado, adaptado à realidade brasileira ${ }^{10} \mathrm{e}$ já testado no contexto cuiabano ${ }^{11}$. Nele fo- ram inseridas as questões referentes ao trabalho, submetidas a um teste-reteste de confiabilidade $^{13}$, com intervalo de 21 dias entre as aplicações. A sua aplicação se deu em sala de aula, sem a presença do professor, por acadêmicos treinados da área de saúde da Universidade Federal de Mato Grosso (UFMT).

O instrumento compôs-se das variáveis:

- Trabalho: Sim/Não;

- Uso recente de: álcool: Sim/Não (abrangendo o consumo de cerveja, chope, vinho, uísque, cachaça, champanhe); tabaco: Sim/Não; outras drogas: Sim/Não (abrangendo o uso de cocaína, maconha, solventes, anfetaminas, barbitúricos, ansiolíticos, opiáceos, antícolinérgicos, xaropes, alucinógenos, orexígenos e anabolizantes); idade do primeiro uso de droga. O termo "uso recente*, com base na Organização Mundial de Saúde $(\mathrm{OMS})^{15}$, refere-se à pessoa que utilizou alguma droga psicoativa pelo menos uma vez nos trinta dias que antecederam a pesquisa.

- Dados sociodemográficas: sexo: Masculino/Feminino; faixa etária: 10-14/ 15-20 anos; nível socioeconômico: baseado em bens de consumo e nível de escolaridade do responsável pela família, conforme os critérios da Associação Brasileira dos Institutos de Pesquisa de Mercado (ABIPEME), cuja soma compreende cinco categorias socioeconômicas $\mathrm{A}+\mathrm{B}+\mathrm{C}+\mathrm{D}+\mathrm{E}$, agrupadas neste estudo em duas categorias: melhor poder aquisitivo $(A+B)$ e médio e baixo poder aquisitivo $(\mathrm{C}+\mathrm{D}+\mathrm{E})$;

- Dados escolares: defasagem escolar: Sim/Não; falta às aulas nos últimos 30 dias que antecederam a pesquisa: (Sim/ Não);

- Dados de comportamento de saúde: prática de esportes categorizada em Sim/Não.

\section{A análise}

O teste do qui-quadrado e de Fisher's 
Exact foram usados para examinar as diferenças entre os dois grupos. Análises bivariadas foram feitas para exploração inicial dos dados, utilizando-se o teste do qui-quadrado para testar a homogeneidade das proporções, em um nível de significância de $0,05 \%$.

Para o controle de variáveis de confusão, utilizou-se um modelo de regressão logística, método stepwise, baseado na razão de verossimilhança, para cálculo das razões oddso (RO) ajustadas. Entraram no modelo todas as variáveis que, na análise bruta, apresentaram valor de $\mathrm{p}$ (significância) igual ou menor que 0,25. Iniciouse por modelo saturado - O teste de Hosmer-Lemeshow ${ }^{16}$ foi usado para avaliar o ajuste do modelo. A presença de confusão foi analisada, retirando-se as co-variáveis, uma a uma, pelo critério de $\mathrm{p}>$ 0,05 e verificando se sua saída não provocava mudanças superiores a 10,0\% dos coeficientes das demais variáveis e, em seguida, comparando-se a nova RO com aquelas obtidas no modelo saturado, permanecendo no modelo final as variáveis com um nível de significância menor ou igual a $0,05 \%$, observado através do teste de Wald.

A entrada e a validação dos dados foram objetivadas pelo programa Epi-info Versão 6.0, em dupla entrada e em arquivos separados, que foram comparados, e corrigidas as diferenças identificadas. Os erros de digitação, que atingiram aproximadamente $1 \%$ em 2.291 questionários, foram corrigidos, e as incoerências identificadas revistas manualmente. A análise crítica dos dados foi realizada conforme metodologia adotada em levantamentos com população estudantil ${ }^{10,17}$. O programa SPSS, Versão 9.0, foi utilizado para a análise estatística.

O presente estudo teve a aprovação da Comissão de Ética em Pesquisa da Universidade Federal de Mato Grosso e do Comitê em Pesquisa da Universidade Federal de São Paulo, Escola Paulista de Medicina, e autorização da Secretaria de Estado de Educação de Mato Grosso.

\section{Resultados}

Nenhum estudante se recusou a participar do estudo, porém foram excluídos $20,3 \%$ por não estarem na faixa etária delimitada, 0,06\% pela resposta positiva à questão fictícia sobre drogas, e $0,89 \%$ por não ter respondido à questão sobre trabalho. Obteve-se as informações de 2.291 estudantes para análise sobre o uso recente de álcool, tabaco e outras drogas, representando $3,7 \%$ do total de estudantes matriculados, o que, na época, apontava para a existência de 61.889 estudantes cursando da $5^{\mathrm{a}}$ série do Ensino Fundamental ao $3^{\circ}$ ano do Ensino Médio. Da amostra mínima requerida para o uso recente (2.472), obteve-se a informação de 798 estudantes adolescentes trabalhadores e 1.493 de não trabalhadores, representando 92,7\% do previsto.

A Tabela 1 apresenta a composição da amostra e dos grupos de estudantes adolescentes trabalhadores e não trabalhadores. O primeiro grupo é formado em sua maioria pelo sexo masculino, insere-se na faixa etária de 15 a 20 anos, falta mais às aulas, apresenta mais defasagem escolar com pouca prática de esportes. $O$ grupo de adolescentes não trabalhadores é formado na maioria pelo sexo feminino, na faixa etária de 10-14 anos. Em ambos os grupos, na sua maioria, as famílias possuem baixo poder aquisitivo (nível $\mathrm{C}$ ) e o responsável tem poucos anos de escolaridade. A maior prevalência de uso recente de álcool (47,4\%, IC 95\% 1,60-2,28), tabaco $(13,6 \%$, IC $95 \% 1,49-2,63)$ e de outras drogas (11,1\%, IC 95\% 1,24-2,26) foi observada entre os estudantes trabalhadores com associação estatisticamente significativa em comparação com os adolescentes não trabalhadores (Figura 1).

Em relação à idade inicial do uso recente de substâncias psicotivas (Figura 2), observou-se que os estudantes adolescentes não trabalhadores apresentaram idade mais precoce, com associação significativa para o primeiro uso de álcool, tabaco e outras drogas, em comparação com os es- 
Tabela 1 - Composição da amostra de estudantes adolescentes trabalhadores e não trabalhadores, segundo características sócio-demográficas, escolares e comportamento de saúde da rede estadual de ensino de Cuiabá-MT, 1998.

Table 1 - Sample of working and non-working adolescent students, according to socialdemographic and, school characteristics and health behavior by the state education network of Cuiabá-MT, 1998

\begin{tabular}{|c|c|c|c|}
\hline Características & $\begin{array}{c}\text { Trabalha } \\
\text { No=798 }(34,8)\end{array}$ & $\begin{array}{l}\text { Não trabalha } \\
\mathrm{N}^{\circ}=1493(65,2)\end{array}$ & $\begin{array}{c}\text { Total } \\
2291(100,0)\end{array}$ \\
\hline \multicolumn{4}{|l|}{ Sexo } \\
\hline Masculino & $422(53,2)$ & $575(38,9)$ & $997(43,9)$ \\
\hline Feminino & $371(46,8)$ & $909(61,1)$ & $1274(56,1)$ \\
\hline Não informado & & & $20(0,9)$ \\
\hline \multicolumn{4}{|c|}{ Faixa etária (anos) } \\
\hline $15-20$ & $599(75,1)$ & $641(42,9)$ & $1240(54,1)$ \\
\hline $10-14$ & $199(24,9)$ & $852(57,1)$ & $1051(45,9)$ \\
\hline \multicolumn{4}{|c|}{ Nível socioeconômico } \\
\hline A & $68(8,5)$ & $88(5,9)$ & $156(6,8)$ \\
\hline B & $149(16,7)$ & $340(22,8)$ & $489(21,3)$ \\
\hline C & $346(43,4)$ & $659(44,1)$ & $1005(43,9)$ \\
\hline D & $174(21,8)$ & $308(20,6)$ & $482(21,0)$ \\
\hline E & $61(7,6)$ & $98(6,6)$ & $159(6,9)$ \\
\hline \multicolumn{4}{|c|}{ Escolaridade responsável } \\
\hline$\leq 8$ anos & $422(52,9)$ & $588(39,4)$ & $1010(44,2)$ \\
\hline$>8$ anos & $229(28,7)$ & $528(35,4)$ & $757(33,0)$ \\
\hline Não sabe & $131(16,4)$ & $358(24,0)$ & $89(21,3)$ \\
\hline Não informou & $16(2,0)$ & $19(1,2)$ & $435(1,5)$ \\
\hline \multicolumn{4}{|l|}{ Faltas às aulas } \\
\hline $\operatorname{Sim}$ & $340(42,6)$ & $473(31,7)$ & $813(35,5)$ \\
\hline Não & $443(55,5)$ & $996(66,7)$ & $1439(62,8)$ \\
\hline Não informado & $15(1,9)$ & $24(1,6)$ & $39(1,7)$ \\
\hline \multicolumn{4}{|c|}{ Defasagem escolar } \\
\hline $\operatorname{Sim}$ & $663(83,1)$ & $949(63,6)$ & $1612(70,4)$ \\
\hline Não & $135(16,9)$ & $544(36,4)$ & $679(29,6)$ \\
\hline \multicolumn{4}{|c|}{ Prática de esportes } \\
\hline Não & $275(34,5)$ & $470(31,5)$ & $745(32,5)$ \\
\hline Sim & $511(64,0)$ & $1011(67,7)$ & $1522(66,4)$ \\
\hline Não informado & $12(1,5)$ & $12(0,8)$ & $24(1,0)$ \\
\hline
\end{tabular}

tudantes trabalhadores (Teste de Tukey, p $<0,05)$.

Na Tabela 2, observa-se que os fatores associados com significância estatística ao uso recente de álcool abrangem os adolescentes trabalhadores do sexo masculino, que pertencem à faixa etária de 15 a 20 anos, cujo chefe da família possui baixa escolaridade, faltam às aulas e estão em defasagem escolar. Embora sem associa- ção significante, a prática de esporte, em sua maioria, foi mais realizada entre os não trabalhadores. Quanto ao uso recente de tabaco, verificaram-se proporções mais elevadas no grupo de trabalhadores na faixa etária de 15 a 20 anos, pertencentes aos níveis socioeconômicos menos favorecidos $(\mathrm{C}+\mathrm{D}+\mathrm{E})$, com diferenças significativas em relação aos não trabalhadores. Observou-se também que o grupo de trabalha- 


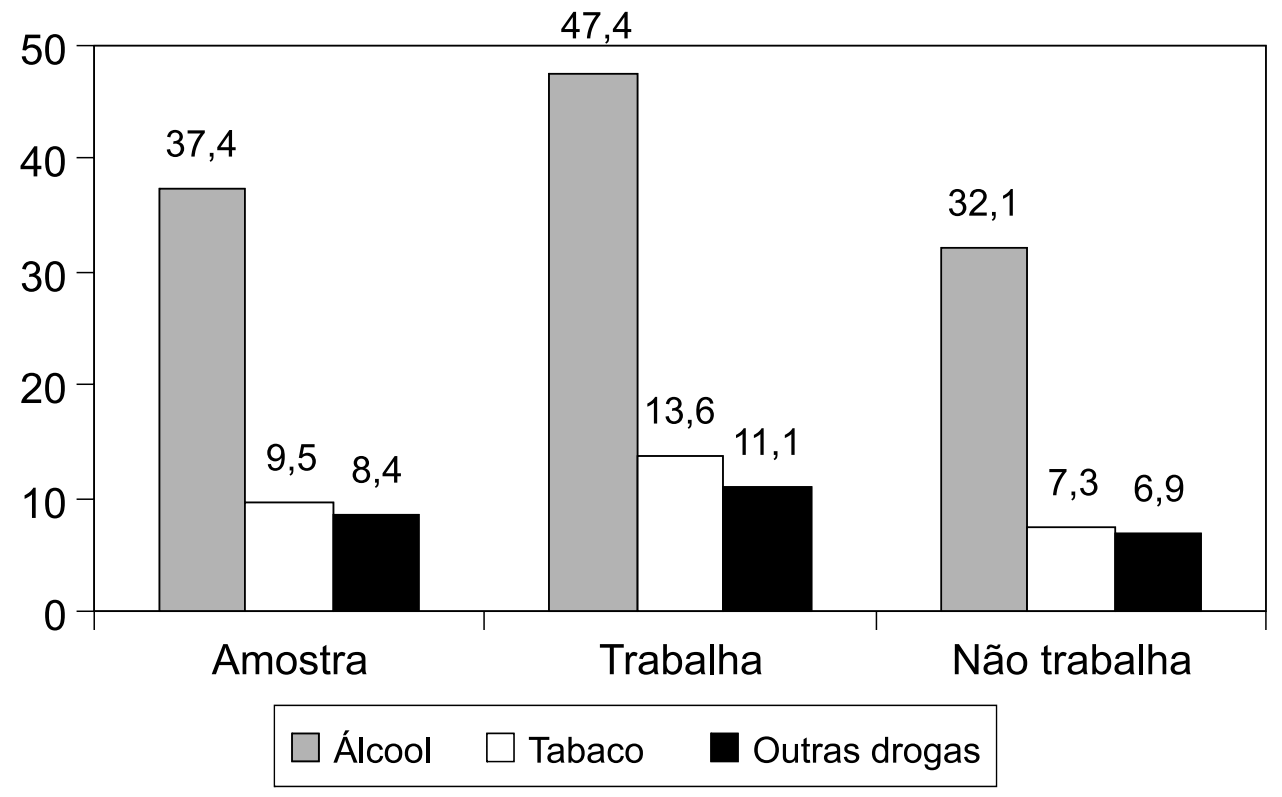

Figura 1 - Prevalência do uso recente de álcool, de tabaco e outras drogas na amostra de estudantes adolescentes e entre trabalhadores e não trabalhadores da rede estadual de ensino de Cuiabá-MT,.1998.

Figure 1 - Prevalence of recent use of alcohol, tobacco and other drugs among working and nonworking adolescent students. Cuiabá-MT, 1998

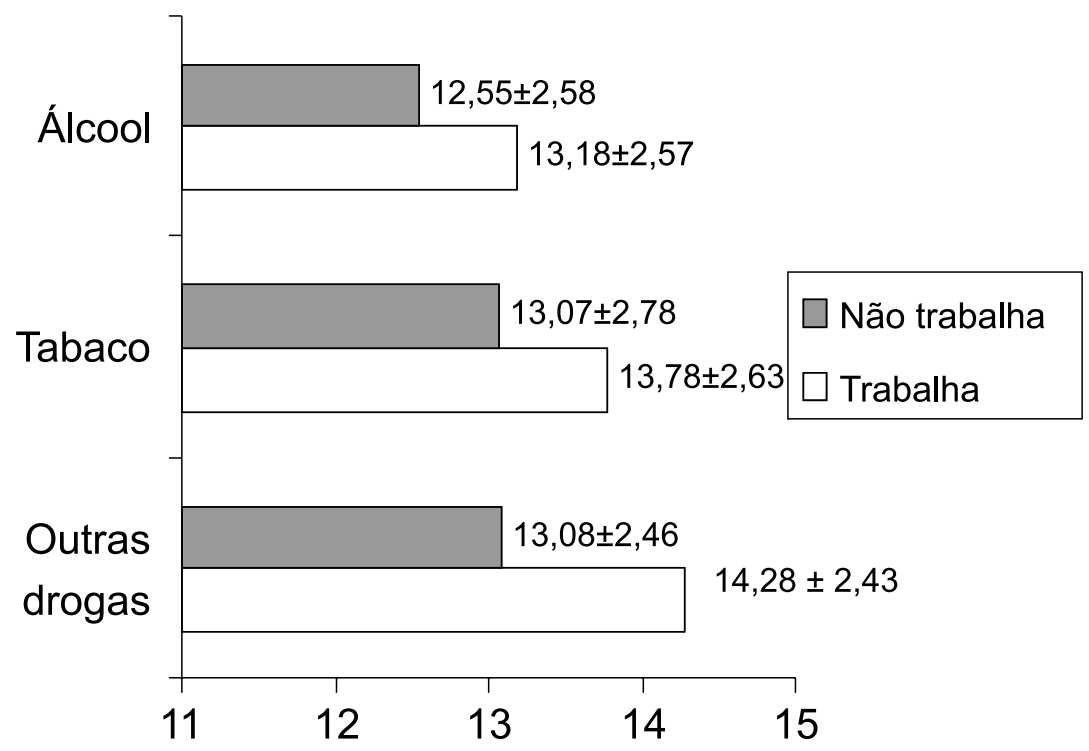

ANOVA seguido do Teste de Tukey, $\mathrm{p}<0,05 /$ ANOVA followed by Tukey Test, $p<0.05$

Figura 2 - Idade inicial do uso de álcool e tabaco e outras drogas entre estudantes adolescentes trabalhadores e não trabalhadores da rede estadual de ensino de Cuiabá-MT, 1998.

Figure 2 - Age of onset of use of alcohol and tobacco and other drugs among working and nonworking adolescent students. Cuiabá, MT, 1998 
dores apresenta as maiores proporções no consumo recente de outras drogas, com associação estatisticamente significativa para aqueles cujo responsável tem baixa escolaridade, aqueles que estão em defasagem escolar e que são do sexo masculino, com idade entre 15 e 20 anos.

Após a análise de regressão logística (Tabela 3), permaneceram no modelo com maior chance de uso recente de substâncias psicoativas os adolescentes trabalhadores na faixa etária de 15 a 20 anos (outras drogas RO = 4,41, IC 95\% 2,20-8,82; álcool RO = 2,56, IC95\% 1,833,57 ; tabaco $\mathrm{RO}=2,45, \mathrm{IC} 95 \% 1,29-4,66$ ), com nível socioeconômico de menor poder aquisitivo (tabaco RO = 2,12, IC95\% 1,15-3,91) e do sexo masculino (álcool RO
$=1,82$, IC95\% 1,36-2,44; outras drogas RO $=1,97$, IC95\% 1,03-3,75), em relação aos não trabalhadores.

Informações adicionais sobre o uso recente de substâncias psicoativas quanto às características do trabalho foram analisadas em relação aos adolescentes não trabalhadores (Tabela 4). Observou-se associação significativa para o uso recente de álcool, tabaco e drogas para os trabalhadores com carga horária diária compreendida entre 4 e 8 horas, que não estão satisfeitos com o trabalho e apresentam outros motivos para trabalhar além apenas ajudar na renda familiar. Trabalhar no setor secundário apresenta maiores chances de uso recente de álcool; no setor terciário, para o uso de tabaco e outras drogas.

Tabela 2 - Uso recente de álcool, tabaco e outras drogas e fatores associados entre estudantes adolescentes trabalhadores e não trabalhadores.Cuiabá-MT, 1998.

Table 2 - Recent use of alcohol, tobacco and other drugs and associated factors among working and non-working adolescent students. Cuiabá-MT, 1998.

\begin{tabular}{|c|c|c|c|c|c|c|c|c|c|}
\hline \multirow[t]{2}{*}{ Variáveis } & \multicolumn{3}{|c|}{$\begin{array}{c}\text { Ácool } \\
\text { Trabalha }\end{array}$} & \multicolumn{3}{|c|}{$\begin{array}{l}\text { Tabaco } \\
\text { Trabalha }\end{array}$} & \multicolumn{3}{|c|}{$\begin{array}{c}\text { Outras Drogas } \\
\text { Trabalha }\end{array}$} \\
\hline & Sim & Não & $\mathrm{RO}_{95 \%}$ & Sim & Não & $\mathrm{RO}_{95 \%}$ & Sim & Não & $\mathrm{RO}_{95 \%}$ \\
\hline \multicolumn{10}{|l|}{ Sexo } \\
\hline Masculino & $208(56,2)^{* *}$ & $191(40,6)$ & $1,88(1,42-2,48)$ & $51(48,1)$ & $42(39,3)$ & $1,43(0,83-2,47)$ & $48(54,5)^{*}$ & $37(36,6)$ & $2,07(1,15-3,71)$ \\
\hline Feminino & $162(43,8)$ & $280(59,4)$ & 1,00 & $55(51,9)$ & $65(60,7)$ & 1,00 & $40(45,5)$ & $64(63,4)$ & 1,00 \\
\hline \multicolumn{10}{|l|}{ Faixa etária } \\
\hline $15-20$ anos & $298(80,1)^{* *}$ & $274(57,9)$ & $2,92(2,13-4,00)$ & $86(81,1)^{* *}$ & $66(61,1)$ & $2,73(1,47-5,09)$ & $71(80,7) * *$ & $47(45,6)$ & $4,97(2,58-9,59)$ \\
\hline 10-14 anos & $74(19,9)$ & $199(42,1)$ & 1,00 & $20(18,9)$ & $42(38,9)$ & 1,00 & $17(19,3)$ & $56(54,4)$ & 1,00 \\
\hline \multicolumn{10}{|l|}{ Nível econômico } \\
\hline$C+D+E$ & $250(67,2)$ & $311(65,8)$ & $1,06(0,80-1,42)$ & $77(72,6)^{*}$ & $61(56,5)$ & $2,04(1,15-3,62)$ & $61(69,3)$ & $62(60,2)$ & $1,49(0,81-2,72)$ \\
\hline$A+B$ & $122(32,8)$ & $162(34,2)$ & 1,00 & $29(38,2)$ & $47(61,8)$ & 1,00 & $27(30,7)$ & $41(39,8)$ & 1,00 \\
\hline \multicolumn{10}{|l|}{ Escol. Responsável } \\
\hline$\leq 8$ anos & $193(52,3) * *$ & $203(46,6)$ & $1,44(0,95-2,17)$ & $61(60,4)$ & $42(39,3)$ & $1,98(0,76-5,25)$ & $44(51,8)^{*}$ & $33(32,7)$ & $3,2(1,25-8,50)$ \\
\hline$>8$ anos & $120(32,5)$ & $178(38,2)$ & $1,41(1,02-1,93)$ & $29(28,7)$ & $50(46,7)$ & $0,79(0,29-2,18)$ & $31(36,5)$ & $44(43,6)$ & $1,69(0,66-4,53)$ \\
\hline não soube informar & $56(15,2)$ & $85(18,2)$ & 1.00 & $11(10,9)$ & $15(14,0)$ & 1,00 & $10(11,8)$ & $24(23,8)$ & 1,00 \\
\hline \multicolumn{10}{|l|}{ Faltas ás aulas } \\
\hline Sim & $196(53,0)^{*}$ & $209(44,6)$ & $1,38(1,05-1,82)$ & $66(64,1)$ & $57(53,3)$ & $1,56(0,90-2,72)$ & $54(62,8)$ & $53(52,0)$ & $1,56(0,86-2,80)$ \\
\hline Não & $174(47,0)$ & $257(55,2)$ & 1,00 & $37(35,9)$ & $50(46,7)$ & 1,00 & $32(37,2)$ & $49(48,0)$ & 1,00 \\
\hline \multicolumn{10}{|l|}{ Defasagem escolar } \\
\hline Sim & $313(84,1)^{* *}$ & $339(71,7)$ & $2,09(1,48-2,95)$ & $91(85,8)$ & $84(77,8)$ & $1,73(0,85-3,52)$ & $77(87,5)^{*}$ & $77(74,0)$ & $2,36(1,09-5,11)$ \\
\hline Não & $59(15,9)$ & $134(28,3)$ & 1,00 & $15(14,2)$ & $24(22,2)$ & 1,00 & $11(12,5)$ & $26(25,2)$ & 1,00 \\
\hline \multicolumn{10}{|l|}{ Prática de esportes } \\
\hline Não & $113(30,5)$ & $133(28,4)$ & $1,10(0,82-1,49)$ & $44(42,3)$ & $34(31,8)$ & $1,57(0,89-2,76)$ & $32(37,2)$ & $28(27,5)$ & $1,56(0,84-2,90)$ \\
\hline Sim & $257(69,5)$ & $335(71,6)$ & 1,00 & $60(57,7)$ & $73(68,2)$ & 1,00 & $54(62,8)$ & $74(72,5)$ & 1,00 \\
\hline
\end{tabular}

OBS. Os valores nas colunas de RO indicam que a categoria foi tomada como referência; $R O=$ Razão de Odds; ${ }^{*} p<0,01 ;{ }^{* *} p<001$ OBS. Values in RO columns indicate that the category was taken as a reference; $R O=$ Reason of Odds; $p<0.01 ;{ }^{*} p<001$ 
Tabela 3 - Modelo de regressão logística para uso recente de álcool, tabaco e outras drogas entre estudantes adolescentes trabalhadores e não-trabalhadores. Cuiabá-MT, 1998.

Table 3 - Adjusted odds ratios model for recent use of alcohol, tobacco and other drugs among working and non-working adolescent students. Cuiabá-MT, 1998

\begin{tabular}{|c|c|c|c|c|c|c|}
\hline \multirow[t]{2}{*}{ Variáveis } & \multicolumn{2}{|c|}{ Alcool } & \multicolumn{2}{|c|}{ Tabaco } & \multicolumn{2}{|c|}{ Outras Drogas } \\
\hline & OR(IC95\%) & Valor $\mathrm{p}$ & OR(IC95\%) & Valor $\mathrm{p}$ & OR(IC95\%) & Valor $p$ \\
\hline \multicolumn{7}{|l|}{ Sexo } \\
\hline Masculino & $1,82(1,36-2,44)$ & 0,0001 & - & - & $1,97(1,03-3,75)$ & 0,03 \\
\hline \multicolumn{7}{|l|}{ Feminino } \\
\hline \multicolumn{7}{|l|}{ Faixa etária } \\
\hline 15-20 anos & $2,56(1,83-3,57)$ & 0,0001 & $2,45(1,29-4,66)$ & 0,06 & $4,41(2,20-8,82)$ & 0,0001 \\
\hline \multicolumn{7}{|c|}{ 10-14 anos } \\
\hline \multicolumn{7}{|c|}{ Nível econômico } \\
\hline$C+D+E$ & - & - & $2,12(1,15-3,91)$ & 0,01 & - & - \\
\hline$A+B$ & & & & & & \\
\hline
\end{tabular}

Tabela 4 - Uso recente de álcool, tabaco e drogas entre estudantes adolescentes trabalhadores, segundo setor produtivo, horas diária, motivação e satisfação do trabalho.Cuiabá-MT, 1998.

Table 4 - Recent use of alcohol, tobacco and drugs among working adolescent students, according to productive sector, daily hour load, motivation and satisfaction at work. Cuiabá-MT, 1998

\begin{tabular}{|c|c|c|c|c|c|c|c|c|c|}
\hline \multirow[t]{2}{*}{ Variáveis } & \multicolumn{3}{|c|}{ Álcool } & \multicolumn{4}{|c|}{ Tabaco } & \multicolumn{2}{|c|}{ Drogas } \\
\hline & Total & $\%$ & RO (IC 95\%) & Total & $\%$ & RO (IC 95\%) & Sim & Não & RO (IC 95\%) \\
\hline \multicolumn{10}{|l|}{ Setor } \\
\hline Não trabalho & 1474 & 32,1 & 1,00 & 1474 & 7,3 & 1,00 & 1491 & 6,9 & 1,00 \\
\hline Secundário & 58 & 55,2 & $2,60(1,48-4,60)^{*}$ & 58 & 12,1 & $1,73(0,64-3,96)$ & 58 & 10,3 & $1,55(0,53-3,73)$ \\
\hline Terciário & 576 & 48,2 & $1,97(1,61-2,41)^{* *}$ & 579 & 13,1 & $1,91(1,38-2,63)^{* *}$ & 586 & 11,6 & $1,76(1,26-2,46)^{* *}$ \\
\hline \multicolumn{10}{|l|}{ Horas diárias } \\
\hline Não trabalha & 1474 & 32,1 & 1,00 & 1474 & 7,3 & 1,00 & 1491 & 6,9 & 1,00 \\
\hline$<4$ horas & 177 & 37,3 & $1,25(0,89-1,75)$ & 178 & 9,0 & $1,24(0,67-2,18)$ & 179 & 11,2 & $1,69(0,96-2,84)$ \\
\hline 4 a 8 horas & 202 & 53,0 & $2,38(1,75-3,24)^{* *}$ & 206 & 15,0 & $2,24(1,40-3,48)^{* *}$ & 208 & 12,5 & $1,92(1,16-3,07)^{*}$ \\
\hline$>8$ horas & 285 & 52,3 & $2,31(1,77-3,02)^{* *}$ & 280 & 14,6 & $2,16(1,43-3,22)^{* *}$ & 285 & 10,5 & $1,58(1,00-2,46)^{*}$ \\
\hline \multicolumn{10}{|l|}{ Motivos } \\
\hline Não trabalho & 1474 & 32,1 & 1,00 & 1474 & 7,3 & 1,00 & 1491 & 6,9 & 1,00 \\
\hline Ajudar renda & 549 & 46,1 & $1,80(1,47-2,22)^{* *}$ & 550 & 12,2 & $1,75(1,25-2,44)^{*}$ & 555 & 10,5 & $1,57(1,10-2,22)^{*}$ \\
\hline Outros & 215 & 50,2 & $2,13(1,58-2,88)^{* *}$ & 212 & 16,0 & $2,41(1,354-3,70)^{* *}$ & 218 & 12,8 & $1,98(1,22-3,13)^{*}$ \\
\hline \multicolumn{10}{|c|}{ Satisfeito/trabalho } \\
\hline Não trabalho & 1474 & 32,1 & 1,00 & 1474 & 7,3 & 1,00 & 1491 & 6,9 & 1,00 \\
\hline Não & 197 & 52,3 & $2,31(1,66-3,16)^{* *}$ & 201 & 13,4 & $1,96(1,20-3,11)^{*}$ & 201 & 14,9 & $2,36(1,47-3,70)^{*}$ \\
\hline Sim & 476 & 46,8 & $1,86(1,50-2,31)^{* *}$ & 472 & 12,5 & $1,80(1,26-2,55)^{* *}$ & 481 & 9,4 & $1,39(0,94-2,02)$ \\
\hline
\end{tabular}

OBS. Os valores 1 nas colunas das RO indicam que a categoria foi tomada como referência; RO: Razão de Odds; ${ }^{*} p<0,01$; ${ }^{* *} p<001$

OBS. Values in RO columns indicate that the category was taken as a reference; $\mathrm{RO}=$ Reason of Odds; $\mathrm{p}<0.01 ;{ }^{*} \mathrm{p}<001$

\section{Discussão}

Nas últimas décadas, os estudos epidemiológicos sobre o uso de drogas com a população estudantil da rede pública no Brasil têm sido relevantes para o conhecimento da magnitude do fenômeno quan- to à prevalência e a fatores associados, subsidiando as políticas públicas de prevenção e tratamento.

No presente estudo, as prevalências do uso recente de álcool, tabaco e outras drogas entre adolescentes trabalhadores e não trabalhadores evidenciam semelhanças $\mathrm{e}$ 
diferenças no comportamento do estudante adolescente de Cuiabá-MT, em relação a outras investigações, mostrando que a questão do uso de substâncias psicoativas entre adolescentes vinculados ou não ao mercado de trabalho é uma questão de saúde pública ${ }^{18-22}$.

Quanto à relação entre trabalho e uso recente de álcool, tabaco e drogas, os dados deste estudo também são consistentes com outro estudo ${ }^{22}$ que verificou maiores prevalências entre os estudantes trabalhadores para o uso de álcool e tabaco com associação estatisticamente significante. Em relação à perda da associação do uso recente de drogas entre os trabalhadores, observou-se semelhança desta investigação com os resultados encontrados em estudo realizado com adolescentes da Tailândia ${ }^{23}$, ou seja, associação do trabalho com o uso de álcool e tabaco entre trabalhadores, em análise bivariada, mas com perda da associação do uso de outras drogas e de sua significância estatística na análise de regressão logística, em comparação com os não trabalhadores.

Várias são as possíveis interpretações sobre a associação do consumo de álcool, tabaco e outras substâncias com o trabalho entre adolescentes ${ }^{20-24}$, pertinentes neste estudo, tais como:

- os jovens em contato com outras pessoas mais velhas no ambiente de trabalho, que usam drogas, apresentam chances maiores para sua iniciação no consumo;

- $\quad$ adolescentes já usuários de substâncias psicoativas, com renda disponível aumentada por mais horas de trabalho, podem encontrar no trabalho uma motivação para apoiar este uso de drogas;

- o trabalho pode ser estressante para os adolescentes, e eles podem tentar aliviar a tensão usando alguma substância psicoativa;

- baixo compromisso com a escola pode ter efeitos em ambas as decisões: trabalhar intensamente e usar alguma substância psicoativa;

- a transição precoce para os papéis de adulto pode ser um mecanismo que conectaria maior atividade de trabalho e uso de alguma substância psicoativa.

As investigações sobre uso de substâncias psicoativas evidenciam que o uso de álcool, de modo geral, inicia-se na adolescência. Os resultados deste estudo mostraram que a média de idade da primeira experimentação do álcool se deu mais precocemente entre os não trabalhadores $(12,55 \pm 2,58)$ do que entre os trabalhadores, seguido pelo tabaco e outras drogas, e se manteve associada à faixa etária de 15 a 20 anos para as três substâncias. É preocupante esta iniciação precoce do uso de drogas lícitas e seus efeitos na saúde mental e física do adolescente, sendo importante que as intervenções se voltem para os aspectos da vulnerabilidade inerente à idade e estabeleçam ações para prevenir o primeiro uso de substâncias psicoativas ${ }^{17}$.

Com relação ao sexo, os homens trabalhadores consumiram mais álcool e outras drogas, não se diferenciando de estudos com população adolescente que também manifestou preferência pelo álcool $^{19,22}$. Também chama a atenção as mulheres com maior uso de tabaco, coadunando-se com estudo realizado mais recentemente com estudantes da rede pública de Cuiabá ${ }^{17}$.

Com relação às condições econômicas da família dos participantes deste estudo, apenas os adolescentes trabalhadores de famílias de níveis menos favorecidos $(\mathrm{C}+\mathrm{D}+\mathrm{E})$ mostraram-se associados ao uso recente de tabaco. Este resultado contrapõe-se aos dados de estudos que evidenciaram associação significativa entre melhor nível socioeconômico e uso de substâncias psicoativas ${ }^{10,17}$, e aos observados por Malcon et al. ${ }^{8}$ para o tabagismo em Pelotas, que não verificaram diferenças significativas entre uso e condições econômicas do adolescente.

Tratando-se da associação de trabalho e uso de substâncias psicoativas com as variáveis escolares, tanto a defasagem como as faltas às aulas estão associadas 
positivamente entre os adolescentes trabalhadores, em análise bivariada. Estes resultados devem ser analisados com cautela, principalmente com a perda de associação no modelo de regressão, embora haja estudos que relacionam o consumo de drogas ao baixo rendimento escolar e à ausência as aulas ${ }^{17,19}$.

Outro resultado que merece atenção refere-se à prática de esportes. Tanto entre os adolescentes trabalhadores como entre os não trabalhadores, observou-se o uso recente de substâncias psicoativas, não havendo diferenças significativas. Estudo realizado com estudantes ${ }^{7}$ também constatou que a prática de esportes não é um fator protetor para coibir o uso de drogas e que infelizmente ainda no seio da sociedade, perpassa a idéia de que manter o adolescente ocupado com atividades extracurriculares o impediria do contato com as drogas?

Embora os resultados do presente estudo sejam consistentes e relevantes, algumas limitações devem ser consideradas, como o delineamento transversal não permitir inferências causais, pois fatores de risco e desfecho são vistos em um mesmo momento, e o viés de causalidade reversa não poder ser eliminado, bem como a não validação do instrumento para investigar comportamento privado e ilegal, como também o viés de informação devido a algum estudante não ter revelado o uso de substâncias por desconfiança ou erro de memória. A comparação com outros estudos fica prejudicada devido a diferenças metodológicas quanto à delimitação do padrão de uso de substâncias psicoativas, do ponto de corte para a carga horária de trabalho e dos critérios para identificação do estudante adolescente trabalhador.

Assim como tem sido mostrado em outros estudos ${ }^{22-24}$, os dados desta pesquisa sugerem que o trabalho está associado ao comportamento do uso recente de álcool, tabaco e drogas entre os estudantes adolescentes de Cuiabá. Entre esses, os mais expostos são os que afirmaram ter uma carga diária de trabalho de 4 a 8 horas, insatisfação com o trabalho, evidenciando que a atividade profissional não impede os adolescentes em idade escolar de beber, fumar e consumir drogas.

Também foi importante o resultado encontrado neste estudo referente ao maior uso recente de álcool entre os adolescentes que trabalham no setor secundário da economia nacional, que abrange, entre outros, o setor da construção civil, área que, além de absorver o maior número de trabalhadores com pouca qualificação profissional, apresentou, em estudo realizado em Cuiabá-MT ${ }^{25}$, proporção elevada de internações por uso de álcool, na faixa etária de 15 a 20 anos. É importante a realização de pesquisas que possam melhor identificar os fatores associados ao consumo de substâncias psicoativas versus segmento profissional.

Desde a coleta dos dados até o momento presente, políticas públicas foram implementadas nas esferas federal, estadual e municipal para a redução do consumo de substâncias psicoativas entre a população estudantil. Porém ainda não se tem a avaliação do seu impacto. Sabe-se apenas que as taxas de agravos de causas externas, especificamente a violência, têm apresentando índices consideráveis de adolescentes, seja como vítimas ou como agressores, sob efeito de alguma substância psicoativa.

Conclui-se que os resultados deste estudo devem ser considerados nas estratégias de prevenção do uso recente de álcool, tabaco e outras drogas na adolescência, e envolver ações conjuntas e articuladas entre as entidades educacionais, de trabalho, família e sociedade como um todo, bem como auxiliar os pesquisadores no aprofundamento da questão do uso indevido de drogas por adolescentes, identificando as diferenças e lacunas no campo da produção do conhecimento. 


\section{Referências}

1. International Labor Organization (ILO). Every child counts: new global estimates on child labour. Geneva International Labour Organization,. 2002.

2. A Atuação do Programa Internacional para a Eliminação do Trabalho Infantil (IPEC) no Brasil. Disponível em: http://www.ilo.org/public/portugue/region/ampro/ brasilia/dezanos/pdf /ipec. Acessado em 15 de março de 2006.

3. Schwartzman S. Trabalho infantil no Brasil-Brasília: OIT. Disponível em: http://www.ilo.org/public/portugue/ region/ampro/brasilia/info/download/trab_inf/ trab_inf.pdf. Acessado em 27 de outubro de 2004.

4. Ministério da Saúde. Transtornos mentais e do comportamento relacionadas ao trabalho.In:Doenças relacionadas ao trabalho: manual de procedimentos para os serviços/Ministério da Saúde do Brasil, Representação no Brasil da Opas/OMS. Elizabeth Costa Dias (Org.). Ministério da Saúde do Brasil/Secretaria de Políticas de Saúde/Departamento de Ações Programáticas Estratégicas/Área técnica de Saúde do Trabalhador. Série A. Normas e Manuais Técnicos; n.114. Brasília DF: 2001.p. 161-191.

5. Soldera M, Dalgalarrondo P, Corrêa Filho HR, Silva, CAM. Uso de drogas psicotrópicas por estudantes: prevalência e fatores sociais associados. Rev Saúde Pública 2004; 38(2): 277-83.

6. Baus J, Kupek E, Pires M. Prevalência e fatores de risco relacionados ao uso de drogas entre escolares. Rev Saúde Pública 2002; 36: 40-6.

7. Carvalho VA, Carlini-Cotrim B. Atividades extracurriculares e prevenção ao abuso de drogas: uma questão polêmica. Rev Saúde Pública 1992; 26(3): 145-9.

8. Malcoln MC, Menezes AMB, Chatkin M. Prevalência e fatores de risco para tabagismo em adolescentes. Rev Saúde Pública 2003; 37 (1): 1-7.

9. Tavares BF, Beria JU, Lima MS. Fatores associados ao uso de drogas entre adolescentes escolares. Rev Saúde Pública 2004; 38(6): 787-96.

10. Galduróz JCF, Nodo AR, Carlini EA. IV levantamento sobre o uso de drogas entre estudantes de $1^{\circ}$. e $2^{\circ}$. graus em 10 capitais brasileiras - 1997. São Paulo: Centro Brasileiro de Informações sobre Drogas Psicotrópicas CEBRID, Departamento de Psicobiologia da Universidade Federal de São Paulo, 1997. p. 130.

11. Souza DPO, Martins DTO O perfil epidemiológico do uso de drogas entre estudantes de $10^{\circ} \mathrm{e} 2 .^{\circ}$ graus da rede estadual de ensino de Cuiabá, 1995. Cad de Saúde Pública 1998; 14: 391-400.

12. Outeiral JO. Adolescer: Estudos sobre adolescência. Porto Alegre: Artes Médicas Sul: 1994. p. 5-9.

13. Souza DPO, Câmara VM; Martins DTO; Valente JG. Confiabilidade do instrumento: construção de questões sobre o trabalho para identificar estudantes adolescentes trabalhadores da rede estadual de ensino de Cuiabá.[CDROM]. VI Congresso de Saúde Coletiva: O sujeito na Saúde Coletiva 2000.

14. Kish L. Survey sampling. Washington; John Wiley \& Sons, 1967. p. 75-142.

15. OMS (Organização Mundial da Saúde) - WHO-World Health Organization Nomenclature and classification of drug and alcohol-related problems: a $\mathrm{WHO}$ Memorandum. Bulletin of the World Health Organization 1981; 59(2): 225-45.

16. Hosmer DW, Lemeshow S. Applied Logistic Regression. New York: John Wiley and Sons; 1989. p. 140-5.

17. Galduróz JCF, Nodo AR, Fonseca AM, Carlini EA. $V$ levantamento sobre o uso de drogas entre estudantes do Ensino Fundamental e Médio da Rede Pública de Ensino nas 27 Capitais Brasileiras - 2004. São Paulo: Centro Brasileiro de Informações sobre Drogas Psicotrópicas CEBRID: Departamento de Psicobiologia da Universidade Federal de São Paulo; 2005; p. 381.

18. Kouvonen A, Lintonen T. Adolescent part-time work and heavy drinking in Finland. Addiction 2002; 97: 311-8.

19. Bachman JG, Schulenberg J. How Part-Time Work Intensity Relates to Drug Use, Problem Behavior, Time Use, and Satisfaction among High School Seniors: Are These Consequences or Merely Correlates? Developmental Psychology 1993; 29: 220-35.

20. McMorris BJ, Uggen C. Alcohol and employment in the transition to adulthood. JHeath Soc Behav2000; 41:276-94.

21. Paschal MJ, Flewelling RL, Russell T. Why is work intensity associated with heavy alcohol use among adolescents? J Adolescent Heath 2004; 34: 79-87.

22. Valois RF, Dunham ACA, Jackson KL, Waller J. Association between employment and substance abuse behaviors among Public High School Adolescents. JAdolescent Heath 1999; 25: 256-63.

23. Wakai K, Miura H,Umenai T. Effect of working status on tobacco, alcohol, and drug use among adolescents in urban area of Thailand. Addictive Behaviors 2005; 30: 457-46.

24. Wu LT, Schlenger WE, Galvin DM. The relationship between employment and substance use among students aged 12 to 17. JAdolescent Health 2003; 32: 5-15.

25. Oliveira MVM. Internações por uso de Álcool entre trabalhadores no Centro de Atendimento Psicossocial Adauto Botelho no município de Cuiabá-MT. Trabalho de Conclusão de Curso (Especialização) - Instituto de Saúde Coletiva, Universidade Federal de Mato Grosso, Cuiabá, 2004.

Recebido em: 25/08/06 Versão final reapresentada em: 06/04/07 Aprovado em: 25/04/07 